OPEN

SUBJECT AREAS:

TWO-DIMENSIONAL

MATERIALS

MATERIALS CHEMISTRY

Received

1 October 2014

Accepted

31 December 2014

Published

27 January 2015

Correspondence and requests for materials should be addressed to

B.C. (bjcho@kims.re.

kr); M.G.H.

(mghahm@kims.re.kr) or D.-H.K. (dhkim2@

kims.re.kr)

* These authors contributed equally to this work.

\title{
Charge-transfer-based Gas Sensing Using Atomic-layer $\mathrm{MoS}_{2}$
}

Byungiin Cho'*, Myung Gwan Hahm*, Minseok Choi², Jongwon Yoon ${ }^{3}$, Ah Ra Kim', Young-Joo Lee', Sung-Gyu Park', Jung-Dae Kwon', Chang Su Kim' ', Myungkwan Song', Yongsoo Jeong ', Kee-Seok Nam', Sangchul Lee ${ }^{5}$, Tae Jin Yoo ${ }^{3}$, Chang Goo Kang ${ }^{6}$, Byoung Hun Lee ${ }^{3}$, Heung Cho Ko ${ }^{3}$, Pulickel M. Ajayan ${ }^{7}$ \& Dong-Ho Kim ${ }^{1}$

\footnotetext{
'Advanced Functional Thin Films Department, Surface Technology Division, Korea Institute of Materials Science (KIMS), 797 Changwondaero, Sungsan-Gu, Changwon, Gyeongnam 642-831, Republic of Korea, ${ }^{2}$ Advanced Characterization and Analysis Group, Korea Institute of Materials Science (KIMS), 797 Changwondaero, Sungsan-Gu, Changwon, Gyeongnam 642-831, Republic of Korea, ${ }^{3}$ School of Materials Science and Engineering, Gwangju Institute of Science and Technology (GIST), 261 Cheomdan-gwagiro, Buk-Gu, Gwangju 500-712, Republic of Korea, ${ }^{4}$ Electrochemistry Department, Korea Institute of Materials Science (KIMS), 797 Changwondaero, Sungsan-Gu, Changwon, Gyeongnam 642-831, Republic of Korea, ${ }^{5}$ Department of Chemical Engineering and Materials Science, Stevens Institute of Technology, Hoboken, New Jersey 07030, United States, ${ }^{6}$ Cambridge Graphene Center, University of Cambridge, $9 \mathrm{JJ}$ Thomson Avenue, Cambridge, United Kingdom, ${ }^{7}$ Department of Materials Science and NanoEngineering, Rice University, 6100 Main Street, Houston, Texas 77005, USA.
}

Two-dimensional (2D) molybdenum disulphide $\left(\mathrm{MoS}_{2}\right)$ atomic layers have a strong potential to be used as 2D electronic sensor components. However, intrinsic synthesis challenges have made this task difficult. In addition, the detection mechanisms for gas molecules are not fully understood. Here, we report a high-performance gas sensor constructed using atomic-layered $\mathrm{MoS}_{2}$ synthesised by chemical vapour deposition (CVD). A highly sensitive and selective gas sensor based on the CVD-synthesised $\mathrm{MoS}_{2}$ was developed. In situ photoluminescence characterisation revealed the charge transfer mechanism between the gas molecules and $\mathrm{MoS}_{2}$, which was validated by theoretical calculations. First-principles density functional theory calculations indicated that $\mathrm{NO}_{2}$ and $\mathrm{NH}_{3}$ molecules have negative adsorption energies (i.e., the adsorption processes are exothermic). Thus, $\mathrm{NO}_{2}$ and $\mathrm{NH}_{3}$ molecules are likely to adsorb onto the surface of the $\mathrm{MoS}_{2}$. The in situ PL characterisation of the changes in the peaks corresponding to charged trions and neutral excitons via gas adsorption processes was used to elucidate the mechanisms of charge transfer between the $\mathrm{MoS}_{2}$ and the gas molecules.

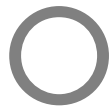

ver the past few decades, metal oxide semiconductors have been applied as conventional chemical sensing materials because of their high sensitivity and relatively low $\cos ^{1-3}$. However, they still have some critical drawbacks. First, metal oxide semiconductors exhibit poor sensitivity and selectivity at room temperature. This obstacle has led to the development of alternative materials such as carbon nanotubes ${ }^{4}$, graphene $^{5}$, and transition metal dichalcogenides (TMDs) ${ }^{6-11}$. Recently, 2D TMDs have attracted much attention for use in next-generation nanoelectronic devices ${ }^{12-14}$, with a single-layer $\mathrm{MoS}_{2}$ transistor having been reported to exhibit outstanding performance ${ }^{15}$. The intrinsic merits of TMDs, including their high surface-to-volume ratio and semiconducting properties, have accelerated the development of a diverse range of applications of these materials as chemical sensors. A recent flurry of research involving $\mathrm{MoS}_{2}$-based gas detection has mitigated the wide chasm between metal oxide materials and alternatives ${ }^{6-11}$. However, the fundamental mechanism of chemical sensing using $\mathrm{MoS}_{2}$ remains unclear, limiting its practical applications. Here, we demonstrate highly sensitive and selective gas detection of $\mathrm{NO}_{2}$ and $\mathrm{NH}_{3}$ using uniform wafer-scale $\mathrm{MoS}_{2}$ nanofilms synthesised by thermal chemical vapour deposition (CVD). We elucidate the charge transfer mechanism of $\mathrm{MoS}_{2}$ gas adsorption using in situ photoluminescence (PL) and computational calculations involving first-principles density functional theory. The peak intensities from the positively charged trions $\left(\mathrm{A}^{+}\right)$and neutral excitons $\left(\mathrm{A}^{0}\right)$ in the PL spectrum show trade-off phenomena by adsorption of each different gas molecule $\left(\mathrm{NO}_{2}\right.$ or $\left.\mathrm{NH}_{3}\right)$ onto the $\mathrm{MoS}_{2}$. The electron depletion of $\mathrm{MoS}_{2}$ by $\mathrm{NO}_{2}$ adsorption leads to an increase in the intensity of the $\mathrm{A}^{+}$peak and a suppression of the intensity of the $\mathrm{A}^{0}$ peak, whereas electron accumulation by $\mathrm{NH}_{3}$ adsorption suppresses the intensity of the $\mathrm{A}^{+}$ peak and increases the intensity of the $\mathrm{A}^{0}$ peak. These in situ PL characterisation results clarify the mechanisms of 
charge transfer between the $\mathrm{MoS}_{2}$ and the gas molecules. These findings will help to implement future gas sensing technologies using diverse two dimensional TMDs nanomaterials.

\section{Results}

Wafer-scale synthesis of atomic-layered $\mathrm{MoS}_{2}$. Most approaches use direct/indirect sulphurisation of Mo-containing thin films to synthesise atomic-layered $\mathrm{MoS}_{2}$ thin films. The precursor is a key factor in the synthesis of $\mathrm{MoS}_{2}$. In previous studies, most authors adopted one of three precursors: molybdenum thin films ${ }^{16}$; molybdenum trioxide ${ }^{17}$; or ammonium thiomolybdate ${ }^{18}$. However, previous methods have involved complex precursor preparations, yielding films with inconsistent quality. In our search for strategies for synthesising uniform wafer-scale $\mathrm{MoS}_{2}$ (see schematic in Fig. 1a), we have focused on the development of a thermal CVD system and process. Atomic-layered $\mathrm{MoS}_{2}$ was grown using molybdenum trioxide $\left(\mathrm{MoO}_{3}\right)$ deposited onto a sapphire substrate and a sulphur powder source. The sublimated sulphur served as a precursor to sulphurise the $\mathrm{MoO}_{3}$ film. To achieve our overall goal of preparing $\mathrm{MoS}_{2}$ films of consistent quality on the desired substrates, we turned our attention to pressure control during the CVD reaction. A recent report indicated that an increase in the amount of either Mo or S atoms results in increased formation of energetically favourable defects on the $\mathrm{MoS}_{2}$ surface during film growth ${ }^{19}$. Thus, we systematically controlled the reaction pressure to provide sufficient sublimated sulphur using a custom-made automatic pressure control system (Supplementary Fig. S1).

The new CVD system design was very effective for the uniform synthesis of $\mathrm{MoS}_{2}$ films on 2-inch sapphire substrates, as illustrated in Fig. 1b. Cross-sectional transmission electron microscopy (TEM) was used to examine the number of layers formed by CVD (Fig. 1c).
The $\mathrm{MoS}_{2}$ films contained double, triple, and, in some cases, more than three layers (additional TEM images, TEM energy-dispersive Xray spectroscopy (EDS) maps, TEM EDS point spectra, atomic force microscopy images, X-ray photoelectron spectra, and absorption spectra are provided in Supplementary Figs. S2-7). The Raman spectrum in Fig. 1d shows the in-plane vibrational mode of the Mo and $S$ atoms $\left(\mathrm{E}_{2 \mathrm{~g}}\right)$ and the out-of-plane vibrational mode of $\mathrm{S}$ atoms $\left(\mathrm{A}_{1 \mathrm{~g}}\right)$ in the as-synthesised $\mathrm{MoS}_{2}$ films ${ }^{20}$. The difference in peak position $(\Delta)$ between the $E_{2 g}$ and $A_{1 g}$ bands, which is a strong indicator of the number of layers, was approximately 22.9. This result indicates that the as-synthesised $\mathrm{MoS}_{2}$ was mainly composed of three layers ${ }^{18,21,22}$. To confirm the wafer-scale synthesis of $\mathrm{MoS}_{2}$, we conducted a largescale structural analysis using Raman mapping and an imaging technique. The Raman mapping area was $50 \times 50 \mu \mathrm{m}^{2}$ with $0.3 \mu \mathrm{m}$ steps (the original Raman mapping spectra are shown in Supplementary Fig. S8). The corresponding Raman images revealed the spatial distribution of $\mathrm{MoS}_{2}$ over a $250-\mu \mathrm{m}^{2}$ area of the substrate (Figs. 1e and $f$ ). The blue and red models show the spatial distributions of the $E_{2 g}$ and $\mathrm{A}_{1 \mathrm{~g}}$ bands, respectively. The as-synthesised $\mathrm{MoS}_{2}$ was highly uniform over a large area of the surface (Figs. 1e and f). Thus, systematic pressure control during the CVD process resulted in highly uniform $\mathrm{MoS}_{2}$ films on the wafer scale.

Gas detection characteristics of the $\mathbf{M o S}_{\mathbf{2}}$ gas sensor. The uniform atomic-layered $\mathrm{MoS}_{2}$ films were used for gas molecule detection (Fig. 2a). Transient resistance responses were investigated using two analyte gases $\left(\mathrm{NO}_{2}\right.$ or $\mathrm{NH}_{3}$ at concentrations from 1.2 to $50 \mathrm{ppm})$. The gas sensitivity was calculated using $\Delta \mathrm{R} / \mathrm{R}_{\mathrm{a}}=\left(\mathrm{R}_{\mathrm{g}}\right.$ $\left.R_{a}\right) / R_{a}$, where $R_{a}$ and $R_{g}$ represent the resistances of the device to air and the analyte gas, respectively. In the $\mathrm{NO}_{2}$ gas mode, the resistance increased (positive sensitivity) (Fig. 2b). The $\mathrm{NO}_{2}$
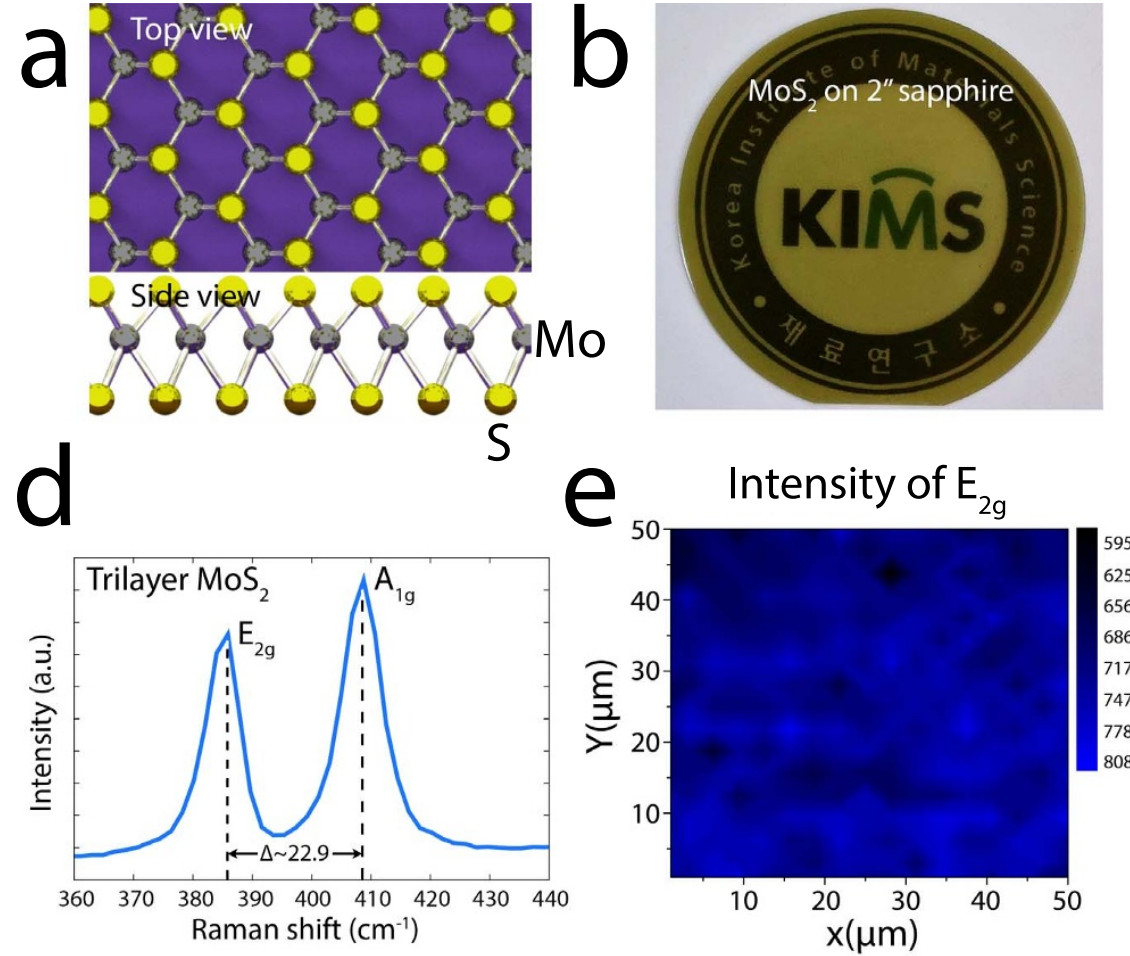
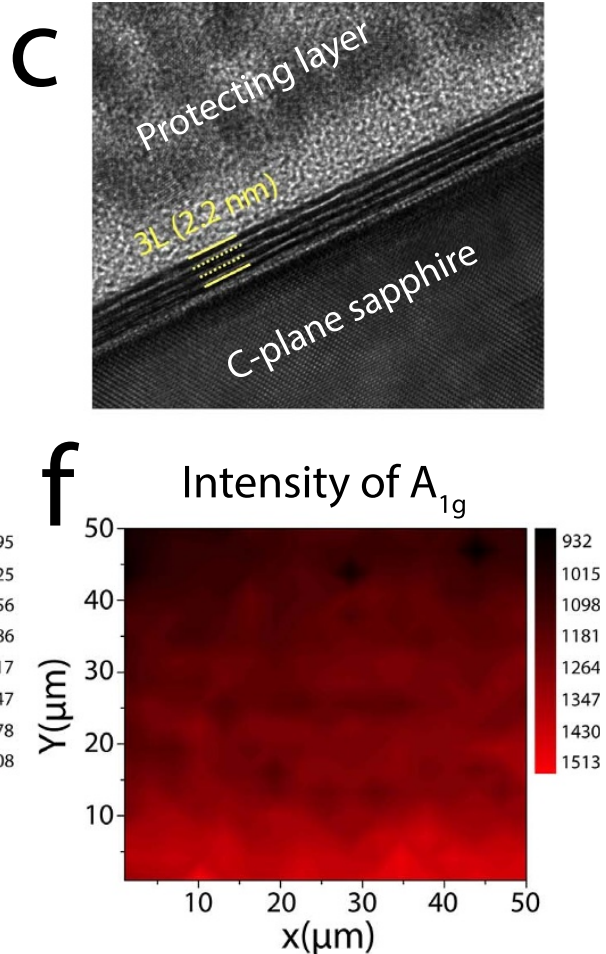

Figure $1 \mid$ Large-scale synthesis of $\mathrm{MoS}_{2}$. (a) Schematic of the atomic-layered $\mathrm{MoS}_{2}$. The quasi-2D MoS $\mathrm{D}_{2}$ was occupied by one Mo (a trigonal prismatic structure) and two $S$ atoms (hexagonal planes). (b) Image of the as-synthesised $\mathrm{MoS}_{2}$ film on the 2-inch sapphire substrate. The as-synthesised $\mathrm{MoS}_{2}$ film was semi-transparent. (c) Cross-sectional TEM images of the as-grown $\mathrm{MoS}_{2}$ films. The image clearly demonstrates that the synthesised $\mathrm{MoS}_{2}$ films consisted of three layers of $\mathrm{MoS}_{2}$. (d) Raman spectrum of the triple-layered $\mathrm{MoS}_{2}$. The spectrum reveals a strong in-plane vibrational mode for the Mo and $\mathrm{S}$ atoms $\left(\mathrm{E}_{2 \mathrm{~g}}\right)$ and an out-of-plane vibrational mode for the $\mathrm{S}$ atoms $\left(\mathrm{A}_{1 \mathrm{~g}}\right)$. The peak position difference $(\Delta)$ between the $\mathrm{E}_{2 \mathrm{~g}}$ and $\mathrm{A}_{1 \mathrm{~g}}$ bands is approximately 22.9, indicating triple-layered $\mathrm{MoS}_{2}$. (e, f) Raman maps of $\mathrm{E}_{2 \mathrm{~g}}$ (blue) and $\mathrm{A}_{1 \mathrm{~g}}$ (red), respectively. The Raman mapping area was $50 \times$ $50 \mu \mathrm{m}^{2}$ with $0.3 \mu \mathrm{m}$ steps. The Raman images show the spatial distribution on the surface of the substrates. 

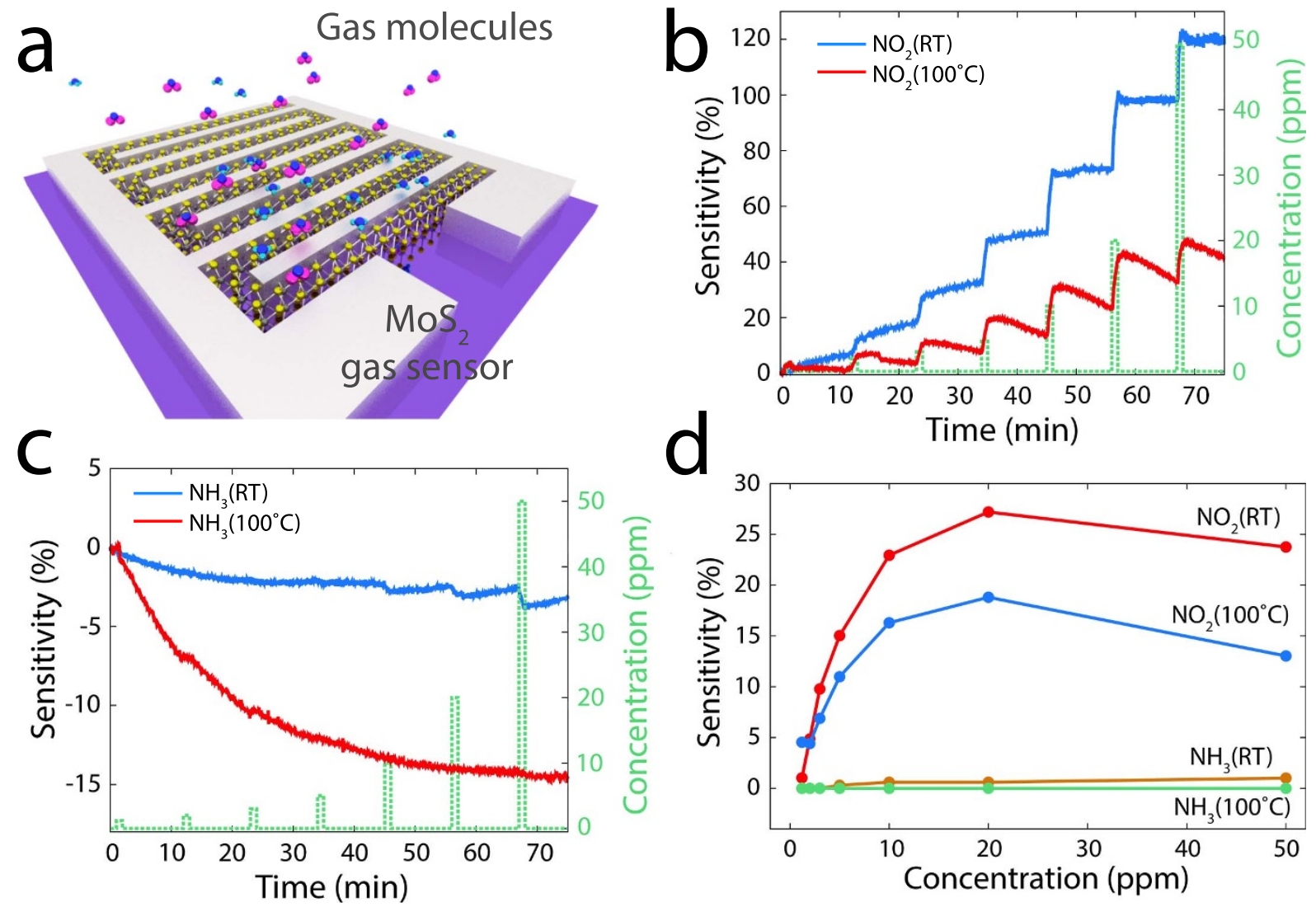

Figure 2 Gas-sensing using the $\mathrm{MoS}_{2}$ device. (a) 3D schematic of the $\mathrm{MoS}_{2}$ gas-sensing device for $\mathrm{NO}_{2}$ and $\mathrm{NH}_{3}$. (b) Transient $\mathrm{NO}_{2}$ gas response at 1.5 to $50 \mathrm{ppm}$ gas at operating temperatures of $\mathrm{RT}$ and $100^{\circ} \mathrm{C}$. In the $\mathrm{NO}_{2}$ gas mode, the resistance increases (positive sensitivity). The recovery rate of $\mathrm{NO}_{2}$ is higher at $100^{\circ} \mathrm{C}$ than at $\mathrm{RT}$. (c) The transient $\mathrm{NH}_{3}$ gas response at 1.5 to $50 \mathrm{ppm}$ gas at operating temperatures of $\mathrm{RT}$ and $100^{\circ} \mathrm{C}$. The resistance decreases with the adsorption of $\mathrm{NH}_{3}$ gas molecules (negative sensitivity). The $\mathrm{NH}_{3}$ sensing signal is negligible at $100^{\circ} \mathrm{C}$. (d) Comparison of the $\mathrm{NO}_{2}$ and $\mathrm{NH}_{3}$ sensitivities at different gas concentrations and operating temperatures. The highest selectivity of $\mathrm{NO}_{2}$ to $\mathrm{NH}_{3}$ was obtained when the concentration reached $20 \mathrm{ppm}$ at $100^{\circ} \mathrm{C}$.

sensitivity values were comparable to those in a previous report ${ }^{8}$. $\mathrm{NO}_{2}$ acts as an electron acceptor, resulting in p-doping (Supplementary Fig. S12). The $\mathrm{NO}_{2}$ molecules on the surface of $\mathrm{MoS}_{2}$ bring the Fermi level closer to the valence-band edge. During the desorption process, thermal energy (heating to $100^{\circ} \mathrm{C}$ ) enhances the rate of desorption of the $\mathrm{NO}_{2}$ molecules from the $\mathrm{MoS}_{2}$ film (Fig. 2b, red line). We next compared the gas sensing characteristics for $\mathrm{NH}_{3}$ to those for $\mathrm{NO}_{2}$ (Fig. 2c). In contrast to the resistance recorded for $\mathrm{NO}_{2}$ molecules, the resistance of the $\mathrm{MoS}_{2}$ sensing device decreased with the adsorption of $\mathrm{NH}_{3}$ gas molecules, i.e., negative sensitivity was observed. $\mathrm{NH}_{3}$ acts as an electron donor (i.e., n-doping) such that it shifts the Fermi level of the $\mathrm{MoS}_{2}$ to the conduction-band edge. However, theoretical calculations indicated that the Fermi-level shift induced by the $\mathrm{NH}_{3}$ molecules is negligible (Supplementary Fig. S12). The measured overall $\mathrm{NH}_{3}$ sensitivities were lower than those of $\mathrm{NO}_{2}$ (Figs. 2b and c) because of the smaller charge transfer of $\mathrm{NH}_{3}$ compared to that of $\mathrm{NO}_{2}{ }^{23}$. The dependence of the gas response on the gas concentration at different operating temperatures is plotted in Fig. 2d. The surface chemical reaction between the $\mathrm{MoS}_{2}$ channel and the $\mathrm{NO}_{2}$ molecules saturated at approximately $20 \mathrm{ppm}$, irrespective of the operating temperature (Fig. 2d, red and blue lines). By contrast, in the case of $\mathrm{NH}_{3}$, the sensitivity at RT gradually increased from 5 to $50 \mathrm{ppm}$ and the sensitivity under $5 \mathrm{ppm}$ was undetectable. However, the sensing signal at $100^{\circ} \mathrm{C}$ was imperceptible at all concentrations (Supplementary Fig. S9). Thus, the recovery rate of $\mathrm{NO}_{2}$ at $100^{\circ} \mathrm{C}$ is clearly superior to that of $\mathrm{NH}_{3}$, which is closely related to the faster desorption process of $\mathrm{NO}_{2}$ molecules as a result of the thermal energy $y^{24,25}$. At $20 \mathrm{ppm}$ and $100^{\circ} \mathrm{C}$, we obtained the best selectivity for $\mathrm{NO}_{2}$ relative to $\mathrm{NH}_{3}(\sim 400 \%$ increase compared to that at RT).

In situ photoluminescence of the $\mathrm{MoS}_{2}$-based gas sensor. To explore the gas adsorption characteristics of the $\mathrm{MoS}_{2}$, we adopted theoretical and experimental approaches. First-principles density functional theory (DFT) calculations were conducted using the screened hybrid functional of Heyd-Scuseria-Ernzerhof with the D2 correction for van der Waals interactions ${ }^{26,27}$ (see the detailed methods in the Supplementary Information). To simulate $\mathrm{NO}_{2}$ and $\mathrm{NH}_{3}$ adsorption onto the $\mathrm{MoS}_{2}$ monolayer, supercells containing 16 Mo and $32 \mathrm{~S}$ atoms with $\mathrm{NO}_{2}$ and $\mathrm{NH}_{3}$ were employed using a $2 \times 2$ $\times 1$ k-point grid. The most stable configurations of $\mathrm{NO}_{2}$ and $\mathrm{NH}_{3}$ reported in a recent study that compared the total energy between different adsorption configurations ${ }^{28}$ were considered. The $\mathrm{NO}_{2}$ and $\mathrm{NH}_{3}$ molecules were preferentially adsorbed onto the top of the hexagon of the $\mathrm{MoS}_{2}{ }^{28}$ (Figs. 3a and b). The adsorption energies of the $\mathrm{NO}_{2}$ and $\mathrm{NH}_{3}$ gas molecules were evaluated using $E_{a}=E_{\left(M_{0 S}-\text { molecule }\right)}-\left[E_{\left(\mathrm{MoS}_{2}\right)}+E_{(\text {molecule })}\right]$, where $E_{\left(\mathrm{MoS}_{2}-\text { molecule }\right)}$ is the total energy of a supercell containing both an $\mathrm{MoS}_{2}$ monolayer and a gas molecule $\left(\mathrm{NO}_{2}\right.$ or $\left.\mathrm{NH}_{3}\right), E_{\left(M o S_{2}\right)}$ is the total energy of the host $\mathrm{MoS}_{2}$ supercell, and $E_{(\text {molecule })}$ is the total energy of a supercell containing a gas molecule. The calculated adsorption energies of $\mathrm{NO}_{2}$ and $\mathrm{NH}_{3}$ were $-0.14 \mathrm{eV}$ and $-0.16 \mathrm{eV}$, respectively. These values were $\sim 0.1 \mathrm{eV}$ smaller than the values obtained using the local density approximation (LDA) because the LDA functional overestimates the adsorption energy ${ }^{28}$. The negative adsorption energies indicate that the adsorption process is exothermic. Thus, 

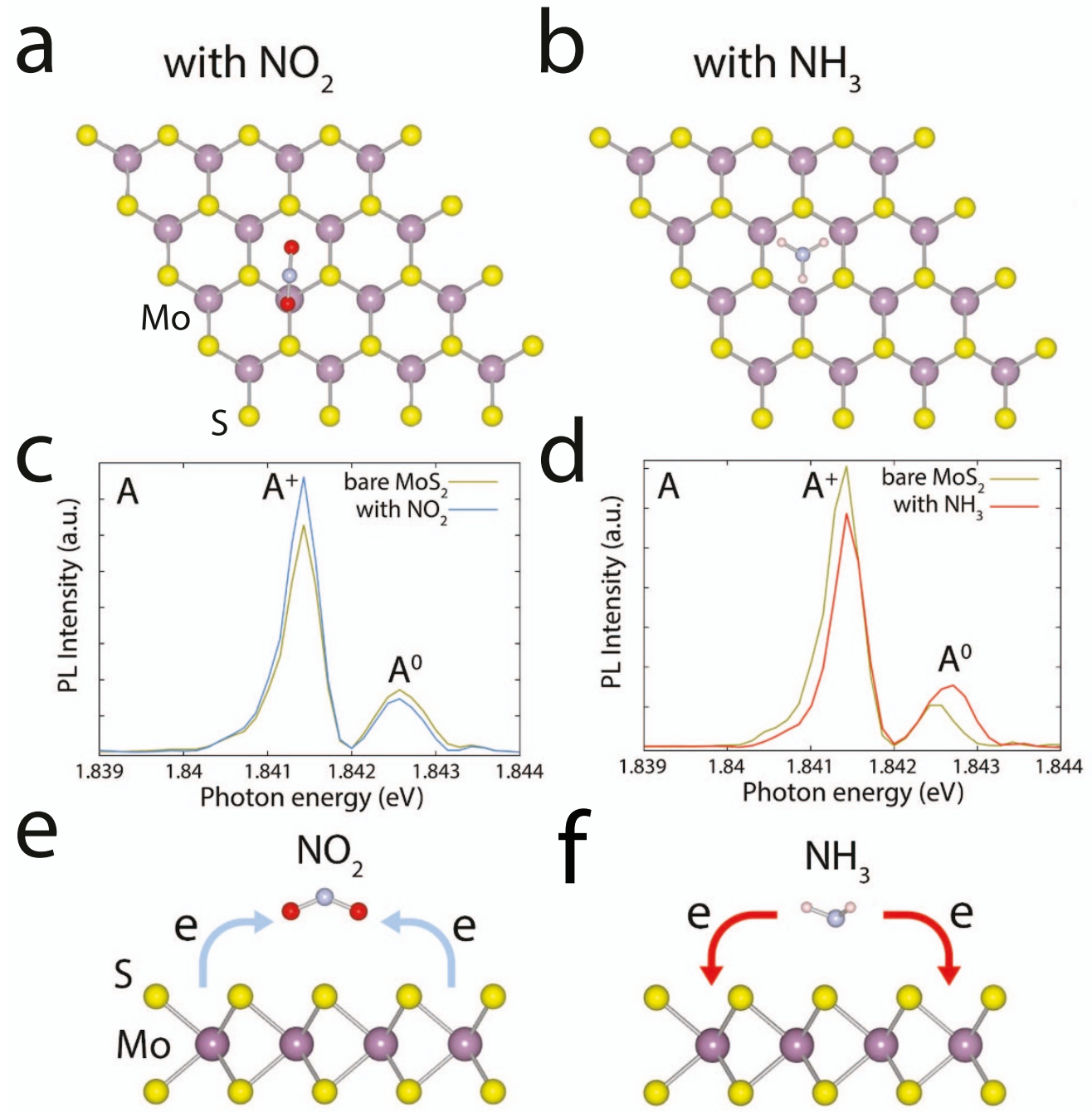

Figure 3 Adsorption configurations and in situ PL. (a, b) Top views of the most favourable configurations for $\mathrm{NO}_{2}(\mathrm{a})$ and $\mathrm{NH}_{3}(\mathrm{~b}) \mathrm{on}_{\mathrm{f}}$ the $\mathrm{MoS}_{2}$. The calculated adsorption energies were $-0.14 \mathrm{eV}$ for $\mathrm{NO}_{2}$ and $-0.16 \mathrm{eV}$ for $\mathrm{NH}_{3}$. The negative adsorption energies indicate that the adsorption process is exothermic, indicating that $\mathrm{NO}_{2}$ and $\mathrm{NH}_{3}$ molecules are likely to be adsorbed onto the surface of the MoS 2 . (c, d) In situ PL spectra recorded from the $\mathrm{MoS}_{2}$ with $\mathrm{NO}_{2}$ (c) and $\mathrm{NH}_{3}$ (d) molecules. The overall intensity of the PL spectra changes in the presence of $\mathrm{NO}_{2}$ and $\mathrm{NH}_{3}$ molecules. The PL intensities of the $\mathrm{A}^{+}$trions and $\mathrm{A}^{0}$ excitons are either suppressed or increased by changes in the concentrations of the charge carriers. (e, $\mathrm{f}$ ) Schematics of the charge density differences for $\mathrm{MoS}_{2}$ in the presence of $\mathrm{NO}_{2}(\mathrm{e})$ and $\mathrm{NH}_{3}$ (f) gas molecules. $\mathrm{NO}_{2}$ molecules on the surface of $\mathrm{MoS}_{2}$ act as electron acceptors, whereas $\mathrm{NH}_{3}$ molecules act as electron donors.

$\mathrm{NO}_{2}$ and $\mathrm{NH}_{3}$ molecules are likely to be adsorbed onto the surface of $\mathrm{MoS}_{2}$.

Next, we turned our attention to the in situ characterisation of PL to study the sensing mechanism in depth. The high temperature during film growth can induce unintentional defects on the substrate/ $\mathrm{MoS}_{2}$ interface. To prevent defects, we transferred the asgrown $\mathrm{MoS}_{2}$ from the sapphire substrate to a $\mathrm{SiO}_{2} / \mathrm{p}+\mathrm{Si}$ substrate. Interestingly, the atomic-layered $\mathrm{MoS}_{2}$ transferred onto the $\mathrm{SiO}_{2} / \mathrm{p}+$ $\mathrm{Si}$ substrate exhibited opposite gas sensitivity (i.e., p-type behaviour) compared to the n-type behaviour of $\mathrm{MoS}_{2}$ on the sapphire substrate (see Supplementary Fig. S13 for details). Dangling bonds at the semiconductor/substrate interface can redefine the effective Fermi levels within gap states, modulating the conductive properties of the $\mathrm{MoS}_{2}{ }^{29}$. The dangling oxygen bonds on the $\mathrm{SiO}_{2}$ surface can result in a p-type $\mathrm{MoS}_{2}$ semiconductor ${ }^{29}$. In the in situ PL characterisation with the two analyte gases (see Supplementary Fig. S10 for details), we observed various intensity changes in the PL spectra for $\mathrm{NO}_{2}$ and $\mathrm{NH}_{3}$ molecules (Figs. $3 \mathrm{c}$ and d). The atomic-layered $\mathrm{MoS}_{2}$ had two main PL peaks associated with the $\mathrm{A}$ and $\mathrm{B}$ excitons $^{30}$ (Supplementary Figs. S11). Spin-orbit coupling-induced valence-band splitting can give rise to $\mathrm{A}$ and $\mathrm{B}$ excitons ${ }^{31}$. After the gas molecules are adsorbed, the PL intensities of the A and B excitons can either be suppressed or increased by changing the concentrations of the charge carriers ${ }^{32,33}$. We here focused on the signal peak of the A exciton. The relatively low-energy A exciton signal expands to two features: a trion of $\mathrm{A}^{-/+}$(two electrons to a hole, resulting in a negatively charged exciton, or an electron to two holes, resulting in a positively charged exciton) and a neutral exciton of $\mathrm{A}^{032,33}$. With the emergence of the trion, we assumed that the exciton is coupled to either another electron or to a hole at the Fermi level. In the case of $\mathrm{MoS}_{2}$, the $\mathrm{A}^{+}$and $\mathrm{A}^{0}$ peaks correspond to the trions $(1.8413 \mathrm{eV})$ and to the neutral excitons $(1.8424 \mathrm{eV})$, respectively. The bare $\mathrm{MoS}_{2}$ notwithstanding, the positive trion (in this case, an electron to two holes, resulting in positively charged excitons, $\mathrm{A}^{+}$) emission dominates the PL spectra because the bare $\mathrm{MoS}_{2}$ on the $\mathrm{SiO}_{2}$ substrate exhibits ptype characteristics, as previously mentioned ${ }^{32}$. As schematically shown in Fig. 3e, the $\mathrm{NO}_{2}$ molecules on the surface of the $\mathrm{MoS}_{2}$ act as electron acceptors (p-type dopants), whereas the $\mathrm{NH}_{3}$ molecules act as electron donors (n-type dopants) ${ }^{28}$. By adsorp- 
tion of $\mathrm{NO}_{2}$ gas molecules, a neutral exciton $\left(\mathrm{A}^{0}\right)$ can be converted into a quasi-particle $\left(\mathrm{A}^{+}\right)$because of excessive holes generated by electron extraction from the $\mathrm{MoS}_{2}{ }^{34}$. As a result, the $\mathrm{A}^{+}$peak in the PL spectrum increases in intensity and the $\mathrm{A}^{0}$ peak is suppressed (Fig. 3c). By contrast, when additional electrons are introduced from the $\mathrm{NH}_{3}$ molecules, the intensity of the $\mathrm{A}^{+}$peak in the PL spectrum is suppressed because of dissociation of the positive trions from the neutral excitons, resulting in increasing neutral excitons $\left(\mathrm{A}^{0}\right)^{35}$, as shown in Fig. $3 \mathrm{~d}$. The in situ PL characterisation clarifies the mechanisms of charge transfer between the $\mathrm{MoS}_{2}$ and the gas molecules.

\section{Discussion}

The gas sensing characteristics of wafer-scale layered $\mathrm{MoS}_{2}$ fabricated by CVD were determined. The gas sensor based on the CVD-fabricated $\mathrm{MoS}_{2}$ exhibited excellent sensitivity and high selectivity. The in situ PL characterisation and theoretical studies elucidated the charge-transfer mechanism between the gas molecules and the $\mathrm{MoS}_{2}$. In-depth PL studies verified that the electron depletion of the $\mathrm{MoS}_{2}$ by $\mathrm{NO}_{2}$ adsorption increased the intensity of the $\mathrm{A}^{+}$peak and suppressed that of the $\mathrm{A}^{0}$ peak, whereas electron accumulation by $\mathrm{NH}_{3}$ adsorption suppressed the intensity of the $\mathrm{A}^{+}$peak and increased that of the $\mathrm{A}^{0}$ peak. Intensive PL characterisation clarified the charge transfer phenomena between the $\mathrm{MoS}_{2}$ and the gas molecules. The results of this study will enable more extensive applications of gas sensing using two dimensional transition metal dichalcogenides nanomaterials.

\section{Methods}

CVD synthesis of the $\mathrm{MoS}_{2}$ nanofilms. $\mathrm{MoS}_{2}$ nanofilms were synthesised using chemical vapour deposition (CVD) (Teraleader Co., Ltd., South Korea) (Supplementary Fig. S1). First, C-plane sapphire substrates were prepared using a typical cleaning process (sonication in acetone, isopropyl alcohol, and deionised water for $10 \mathrm{~min}$ each). $\mathrm{MoO}_{3}$ films $(5 \mathrm{~nm})$ were deposited onto the clean substrates using a thermal evaporator. The pre-deposited $\mathrm{MoO}_{3}$ samples were placed at the centre of the furnace, and $\sim 1 \mathrm{~g}$ of sulphur powder, which was used as a sulphur precursor, was subsequently loaded into a quartz boat in an independently temperature-controllable flange heater located near the inlet of the furnace. The furnace and the flange heater were heated to $\sim 850^{\circ} \mathrm{C}$ and $\sim 180^{\circ} \mathrm{C}$, respectively, for $1 \mathrm{~h}$. The process was maintained for an additional $1 \mathrm{~h}$ under flowing $\mathrm{Ar} / \mathrm{H}_{2}$ gas (volume ratio: $\mathrm{Ar}: \mathrm{H}_{2}=85: 15 \%$ ) at a chamber pressure of 760 torr. The $\mathrm{MoO}_{3}$ film was converted into a $\mathrm{MoS}_{2}$ nanofilm via a two-step reaction (the reduction of $\mathrm{MoO}_{3}$ by hydrogen gas, followed by sulphurisation of the reduced $\mathrm{MoO}_{3}$ with sublimated sulphur gases). Finally, the furnace was rapidly cooled to room temperature by opening the chamber box after the furnace was turned off.

Fabrication of the $\mathrm{MoS}_{2}$ sensing device. The $\mathrm{SiO}_{2}(300 \mathrm{~nm}) / \mathrm{C}$-plane sapphire was cleaned using a typical cleaning process (sequential sonication in acetone, isopropyl alcohol, and deionised water for $10 \mathrm{~min}$ each). $\mathrm{A} \mathrm{MoO}_{3}$ film $(\sim 5 \mathrm{~nm}$ thick) was patterned with an active shadow mask using a thermal evaporator. The patterned $\mathrm{MoO}_{3}$ film was converted into a $\mathrm{MoS}_{2}$ nanofilm by CVD. Using a thermal evaporator and a shadow mask with an interdigitated electrode array structure consisting of two opposing comb-shaped electrodes with a width of $400 \mu \mathrm{m}$ and a gap of $100 \mu \mathrm{m}$, we deposited an Ag film (100 nm; used for the electrodes) onto the $\mathrm{MoS}_{2}$ nanofilm. Detailed fabrication schemes are provided in Supplementary Fig. S14.

Characterisation of CVD-synthesised $\mathrm{MoS}_{2}$, gas sensing, DFT calculations, in situ PL tests. See the detailed methods in the Supplementary Information.

1. Fine, G. F., Cavanagh, L. M., Afonja, A. \& Binions, R. Metal oxide semi-conductor gas sensors in environmental monitoring. Sensors (Basel). 10, 5469-5502 (2010).

2. Pearton, S. J. et al. Recent advances in wide bandgap semiconductor biological and gas sensors. Prog. Mater. Sci. 55, 1-59 (2010).

3. Huang, J. \& Wan, Q. Gas sensors based on semiconducting metal oxide onedimensional nanostructures. Sensors (Basel). 9, 9903-9924 (2009).

4. Kong, J. Nanotube Molecular Wires as Chemical Sensors. Sience 287, 622-625 (2000).

5. Schedin, F. et al. Detection of individual gas molecules adsorbed on graphene. Nat. Mater. 6, 652-655 (2007).

6. Late, D. J. et al. Sensing Behavior of Atomically Thin-Layered $\mathrm{MoS}_{2}$ Transistors. ACS Nano 7, 4879-4891 (2013).

7. Li, H. et al. Fabrication of single- and multilayer $\mathrm{MoS}_{2}$ film-based field-effect transistors for sensing NO at room temperature. Small 8, 63-67 (2012).
8. He, Q. et al. Fabrication of flexible $\mathrm{MoS}_{2}$ thin-film transistor arrays for practical gas-sensing applications. Small 8, 2994-2999 (2012).

9. Lee, K., Gatensby, R., McEvoy, N., Hallam, T. \& Duesberg, G. S. High Performance Sensors Based on Molybdenum Disulfide Thin Films. Adv. Mater. 25, 6699-6702 (2013).

10. Yao, Y. et al. High-concentration aqueous dispersions of $\mathrm{MoS}_{2}$. Adv. Funct. Mater. 23, 3577-3583 (2013).

11. Perkins, F. K. et al. Chemical vapor sensing with monolayer $\mathrm{MoS}_{2}$. Nano Lett. 13, 668-673 (2013)

12. Wang, Q. H., Kalantar-Zadeh, K., Kis, A., Coleman, J. N. \& Strano, M. S. Electronics and optoelectronics of two-dimensional transition metal dichalcogenides. Nat. Nanotechnol. 7, 699-712 (2012).

13. Ganatra, R. \& Zhang, Q. Few-Layer $\mathrm{MoS}_{2}$ : A Promising Layered Semiconductor. ACS Nano 8, 4074-4099 (2014).

14. Jariwala, D., Sangwan, V. K., Lauhon, L. J., Marks, T. J. \& Hersam, M. C. Emerging Device Applications for Semiconducting Two-Dimensional Transition Metal Dichalcogenides. ACS Nano 8, 1102-1120 (2014).

15. Radisavljevic, B., Radenovic, A., Brivio, J., Giacometti, V. \& Kis, A. Single-layer $\mathrm{MoS}_{2}$ transistors. Nat. Nanotechnol. 6, 147-150 (2011).

16. Lee, Y. et al. Synthesis of wafer-scale uniform molybdenum disulfide films with control over the layer number using a gas phase sulfur precursor. Nanoscale 6 , 2821-2826 (2014).

17. Lin, Y.-C. et al. Wafer-scale $\mathrm{MoS}_{2}$ thin layers prepared by $\mathrm{MoO}_{3}$ sulfurization. Nanoscale 4, 6637-6641 (2012).

18. Liu, K.-K. et al. Growth of large-area and highly crystalline $\mathrm{MoS}_{2}$ thin layers on insulating substrates. Nano Lett. 12, 1538-1544 (2012).

19. Najmaei, S. et al. Vapour phase growth and grain boundary structure of molybdenum disulphide atomic layers. Nat. Mater. 12, 754-759 (2013).

20. Bertrand, P. A. Surface-phonon dispersion of $\mathrm{MoS}_{2}$. Phys. Rev. B 44, 5745-5749 (1991).

21. Li, S. et al. Quantitative raman spectrum and reliable thickness identification for atomic layers on insulating substrates. ACS Nano 6, 7381-7388 (2012).

22. Liu, Y. et al. Layer-by-layer thinning of $\mathrm{MoS}_{2}$ by plasma. ACS Nano 7, 4202-4209 (2013).

23. Yue, Q., Shao, Z., Chang, S. \& Li, J. Adsorption of gas molecules on monolayer $\mathrm{MoS}_{2}$ and effect of applied electric field. Nanoscale Res. Lett. 8, 425 (2013).

24. Yavari, F. et al. High sensitivity gas detection using a macroscopic threedimensional graphene foam network. Sci. Rep. 1, 166 (2011).

25. Choi, H. et al. Flexible and transparent gas molecule sensor integrated with sensing and heating graphene layers. Small 10, 3812 (2014).

26. Heyd, J., Scuseria, G. E. \& Ernzerhof, M. Hybrid functionals based on a screened Coulomb potential. J. Chem. Phys. 118, 8207-8215 (2003).

27. Krukau, A. V., Vydrov, O. a., Izmaylov, A. F. \& Scuseria, G. E. Influence of the exchange screening parameter on the performance of screened hybrid functionals. J. Chem. Phys. 125, 224106 (2006)

28. Yue, Q., Shao, Z., Chang, S. \& Li, J. Adsorption of gas molecules on monolayer $\mathrm{MoS}_{2}$ and effect of applied electric field. Nanoscale Res. Lett. 8, 425 (2013).

29. Dolui, K., Rungger, I. \& Sanvito, S. Origin of the n-type and p-type conductivity of $\mathrm{MoS}_{2}$ monolayers on a $\mathrm{SiO}_{2}$ substrate. Phys. Rev. B 87, 165402 (2013).

30. Splendiani, A. et al. Emerging photoluminescence in monolayer $\mathrm{MoS}_{2}$. Nano Lett. 10, 1271-1275 (2010)

31. Coehoorn, R., Haas, C. \& de Groot, R. A. Electronic structure of $\mathrm{MoSe}_{2}, \mathrm{MoS}_{2}$, and $\mathrm{WSe}_{2}$. II. The nature of the optical band gaps. Phys. Rev. B 35, 6203-6206 (1987).

32. Mak, K. F. et al. Tightly bound trions in monolayer $\mathrm{MoS}_{2}$. Nat. Mater. 12, 207-211 (2013).

33. Mouri, S., Miyauchi, Y. \& Matsuda, K. Tunable photoluminescence of monolayer $\mathrm{MoS}_{2}$ via chemical doping. Nano Lett. 13, 5944-5948 (2013).

34. Ross, J. S. et al. Electrical control of neutral and charged excitons in a monolayer semiconductor. Nat. Commun. 4, 1474 (2013).

35. Mao, N., Chen, Y., Liu, D., Zhang, J. \& Xie, L. Solvatochromic effect on the photoluminescence of $\mathrm{MoS}_{2}$, monolayers. Small 9, 1312-1315 (2013).

\section{Acknowledgments}

This study was supported financially by the Fundamental Research Program (PNK3770 and PNK4060) of the Korean Institute of Materials Science (KIMS) and by the "Gyeongsangnam, Changwon Science Research Park Project" of the Grant of the Korean Ministry of Science, ICT and Future Planning. M. G. H. and B. C. are grateful for support from the Basic Science Research Program of the National Research Foundation of Korea (NRF) funded by the Ministry of Science, ICT \& Future Planning

(NRF-2014R1A1A1006214 and NRF-2014R1A1A1036139). MC was supported by Global Frontier Program through the Global Frontier Hybrid Interface Materials (GFHIM) of the National Research Foundation of Korea (NRF) funded by the Ministry of Science, ICT \& Future Planning (2013M3A6B1078872).

\section{Author contributions}

B.C., M.G.H. and D.-H.K. designed and supervised the experiments. B.C., M.G.H. and A.R.K. synthesised the $\mathrm{MoS}_{2}$. B.C., M.G.H., J.Y., A.R.K. and Y.-J.L. characterised the $\mathrm{MoS}_{2}$. B.C. and A.R.K. fabricated the $\mathrm{MoS}_{2}$-based devices. B.C., M.G.H., S.L., T.J.Y. and C.G.K. measured the $\mathrm{MoS}_{2}$ sensing devices. M.G.H. and M.C. performed the first-principles DFT 
calculations for the $\mathrm{MoS}_{2}$ with gas molecules. B.C., M.G.H., M.C., S.-G.P., J.-D.K., C.S.K., M.S., Y.J., K.-S.N., B.H.L., H.C.K., P.M.A. and D.-H.K. analysed the data. B.C., M.G.H. and D.-H.K. co-wrote the paper. All authors discussed the results and commented on the manuscript.

\section{Additional information}

Supplementary information accompanies this paper at http://www.nature.com/ scientificreports

Competing financial interests: The authors declare no competing financial interests.
How to cite this article: Cho, B. et al. Charge-transfer-based Gas Sensing Using Atomic-layer $\mathrm{MoS}_{2}$. Sci. Rep. 5, 8052; DOI:10.1038/srep08052 (2015).

(c) $\Theta$ This work is licensed under a Creative Commons Attribution-NonCommercialNoDerivs 4.0 International License. The images or other third party material in this article are included in the article's Creative Commons license, unless indicated otherwise in the credit line; if the material is not included under the Creative Commons license, users will need to obtain permission from the license holder in order to reproduce the material. To view a copy of this license, visit http:// creativecommons.org/licenses/by-nc-nd/4.0/ 